\title{
Apoyo social, clima social y percepción de conflictos en un contexto educativo intercultural
}

\author{
Isabel Hombrados-Mendieta* y Margarita Castro-Travé
}

Universidad de Málaga, España

\begin{abstract}
Resumen: Este trabajo pretende estudiar las relaciones de apoyo social del alumnado en un contexto escolar intercultural, conocer el apoyo social recibido por parte de los iguales y el profesorado y comprobar si el apoyo social se relaciona positivamente con el clima social del centro escolar y la disminución de conflictos en el aula. La muestra está constituida por 512 estudiantes de centros educativos de la provincia de Málaga, donde conviven estudiantes autóctonos, inmigrantes y de etnia gitana de edades comprendidas entre los 12 y los 17 años. Los resultados indican diferencias significativas entre los estudiantes según su procedencia. El apoyo social proporcionado por los estudiantes autóctonos e inmigrantes se relaciona positivamente con el clima social y negativamente con los problemas de convivencia en el aula y con los conflictos debidos a las diferencias étnicas/culturales, aunque estas relaciones no han sido significativas para los estudiantes de etnia gitana. El apoyo proporcionado por el profesorado también correlaciona significativamente con el clima social del centro y la disminución de problemas de convivencia en el aula pero escasamente con la disminución de los conflictos debidos a las diferencias culturales.

Palabras clave: Apoyo social; clima social; convivencia intercultural; percepción de conflictos.
\end{abstract}

\section{Introducción}

Actualmente la educación en nuestro país está abordando uno de los retos más importantes que se le presentan: el fenómeno de la inclusión de un alumnado cuya diversidad cultural no era antes conocida (Esteve, 2003). Según el Centro de Investigación y Documentación Educativa del Ministerio de Educación (2007), en el curso escolar 1994/1995, el alumnado inmigrante se situaba en 53213 estudiantes, mientras que en el curso 2006/2007, eran ya 591560, representando un $8.35 \%$ de la población total de alumnado escolarizado en las enseñanzas de régimen general no universitarias. Aunque la incorporación de inmigrantes a las aulas se ha ralentizado en los últimos años hay que destacar según el informe Datos y Cifras del Ministerio de Educación (2010) que durante el curso 2008/09 el alumnado extranjero supone un $9.7 \%$ del total. Dentro de Andalucía son Málaga y Almería las dos provincias andaluzas con más alumnado extranjero en el curso 2009/10 (Observatorio Permanente Andaluz de las Migraciones, 2010).

Si bien este porcentaje todavía no es tan significativo como en otros países de nuestro entorno europeo, sí es relevante destacar la rapidez en el crecimiento del número de estudiantes de origen extranjero que está llegando a nuestro sistema educativo (Leiva, 2007). El alumnado procedente de América del Sur es el más numeroso en el sistema educativo español, seguido por el alumnado procedente de África. Ac-

* Dirección para correspondencia [Correspondence address]: Isabel Hombrados-Mendieta. Dpto. de psicología social. Facultad de Psicología, Universidad de Málaga, Campus de Teatinos S/N, 29071 Málaga (España). E-mail: mihombrados@uma.es
Title: Social support, social climate, and the perception of conflict in an intercultural educational context.

Abstract: This study investigates social support among students in an intercultural school context, the social support received from peers and teachers, and assesses whether social support is positively associated with the social climate at school and reduced conflict in the classroom. The sample consisted of 512 students from schools in the province of Malaga, Spain, and included native Spanish, immigrant and Spanish gipsy students aged between 12 years and 17 years. The results indicate significant differences between the students depending on their origin. Social support provided by the native and immigrant students was positively associated with the social climate, but was negatively associated with the problems of being together in the classroom and with conflict due to ethnic and cultural differences. These associations did not reach statistical significance in relation to the Spanish gipsy students. Support provided by teachers also significantly correlated with the social climate at the school and with the reduction in problems of being together in the classroom, but barely correlated with the reduction in intercultural conflict.

Key words: Social support; social climate; intercultural coexistence; perception of conflict.

tualmente ha aumentado significativamente el número de estudiantes procedentes del resto de Europa (Ministerio de Educación y Ciencia, 2007).

En nuestro país, los flujos migratorios tienen cada vez más un carácter familiar por el incremento de las reagrupaciones y la formación de familias en destino. El impacto de la inmigración en las relaciones sociales intrafamiliares es compleja ya que la inmigración puede implicar separaciones y reunificaciones familiares, así como la transformación de roles en la familia durante el proceso de adaptación a la vida en un nuevo país (Suárez-Orozco, 2001). La inmigración también puede afectar a las relaciones que los jóvenes mantienen con los iguales y el profesorado. Esto significa que la inmigración y la integración de los inmigrantes se extienden a otros ámbitos de sociabilidad como la escuela. Existen algunos problemas que afectan directamente a los adolescentes de origen extranjero que viven hoy en España, por ejemplo, el de enfrentarse a la percepción social que se tiene del inmigrante, o en el plano de las relaciones sociales, a la comunicación con sus iguales, en la escuela o en el lugar de residencia, "dado que los patrones de relación social pueden diferir sustancialmente de los de sus pares autóctonos, incorporándose elementos nuevos en relación con lo racial, lo lingüístico y lo religioso" (Giménez y Pérez, 2003, p. 423).

Si bien el fenómeno de la inmigración es reciente, en el contexto escolar no lo es la presencia de grupos minoritarios como los estudiantes de etnia gitana. Sin embargo, son escasos los estudios que profundizan en este colectivo. Según Andrés (2006), actualmente la situación educativa del alumnado gitano respecto a los niveles de asistencia, es que en torno al $80 \%$ de este alumnado que comienza $1^{\circ}$ de la ESO abandona la etapa antes de acabar el último curso. Por otra 
parte, aunque existe menor matriculación en el caso de las chicas gitanas que en el de los chicos al iniciar la nueva eta$\mathrm{pa}$, las primeras tienen menos índices de abandono prematuro en el segundo ciclo de la ESO. Un indicador importante que nos informa acerca de su nivel de incorporación escolar es el grado de interacción entre iguales, ya que las relaciones que un estudiante establece en el aula es un fiel reflejo de la capacidad del sistema educativo para conseguir que la comunidad escolar sea participativa e integradora. Los resultados de algunas investigaciones (Abajo, 2004; Fernández y Soriano, 1996; León, 2008) concluyen que existe un bajo estatus social del alumnado gitano entre los compañeros/as del grupo mayoritario autóctono. Asimismo, aunque la interacción social con el profesorado suele ser positiva, parece existir un pequeño porcentaje de alumnado con respecto al cual el profesorado tiene bajas expectativas académicas.

Por tanto, el enriquecimiento mutuo que supone el contacto con otras culturas y costumbres es uno de los objetivos de la educación intercultural. Hoy se habla de la importancia de crear escuelas interculturales, donde se respeten las diferencias culturales y étnicas y todos los estudiantes sean tratados en un plano de igualdad. Para ello se han de potenciar las relaciones interpersonales entre todos los miembros de la comunidad educativa, lo que contribuirá a mejorar el clima social del centro escolar. La educación intercultural se plantea como una propuesta de acción educativa donde prevalece el reconocimiento de la existencia de los otros como sujetos poseedores de una cultura diferente y el conocimiento de lo que esto significa en términos de semejanzas y diferencias con la propia cultura escolar, caracterizada por múltiples influencias (Díaz-Aguado, 2003). La mayoría de autores y especialistas en educación intercultural (Aguado, 2003; Essomba, 2007; Jordán, 1999, 2007; Santos, 2008; Soriano, 2008) coinciden en destacar que los conflictos derivados del multiculturalismo escolar pueden ser positivos para la propia escuela, a semejanza de lo que ocurre en el ámbito social, ya que se constituyen como claves para la mejora en las relaciones interpersonales siempre que los principios que guíen el diálogo sean el respeto, y el reconocimiento del otro en su diferencia (Bartolomé, 2002; López, 2006). La sociedad actual no es homogénea, en su seno conviven muchas culturas que están siendo representadas, a través de los niños gitanos y de los hijos de inmigrantes que conviven actualmente con el resto de ciudadanos del país. Los adolescentes viven hoy en un entorno donde la presencia de la diversidad cultural es una realidad cotidiana. Sin embargo, esta convivencia multicultural puede ser fuente de conflictos en el aula. El conflicto escolar multicultural haría referencia a toda situación escolar donde acontece una divergencia entre miembros de diferentes grupos culturales que conviven en un centro educativo por cuestiones de índole cultural (por ejemplo incomunicación entre familias y entre alumnos de diferentes culturas y etnias, malentendidos por el empleo de códigos de referencia cultural distinta), o también a una situación de desequilibrio derivada de una compleja red de significados afectivos, emotivos, políticos, éticos - definidos de forma implícita (Leiva, 2007). Hay que señalar que los conflictos que se dan en los contextos educativos donde conviven diferentes culturas tienen unas características cuyo análisis y estudio han estado, generalmente, determinados por la reflexión de los distintos modelos comunicativos, de disciplina y de clima de aula. En este sentido, para Jordán (2007), es necesario indagar en la cultura del diálogo y de la participación, de tal manera que la participación del alumnado en la resolución de los conflictos influirá positivamente en la convivencia y en el clima escolar.

Actualmente, se considera que un clima social adecuado en el centro escolar favorece la integración social de los estudiantes (Trianes, 2000). Precisamente por ello, una de las variables más importantes en la creación de ese clima positivo es la existencia de unas relaciones sociales adecuadas y la percepción de apoyo social entre los miembros de la red. La teoría de Durkheim (1976) de la integración social sugiere que la interacción y el apego con los otros pueden tener consecuencias importantes y beneficiosas durante toda la vida. Las personas que crean y mantienen estrechas relaciones con la familia, los amigos y los vecinos experimentan los beneficios positivos y duraderos de esta integración social (Lin, 2001; Sarason, Pierce y Sarason, 1990). La investigación empírica tiende a mostrar consistentemente que el apoyo social es un predictor importante del bienestar psicológico de los adultos (Harknett, 2006; Henly, Danziger y Offer, 2005; Thoits, 1995) y de los niños y jóvenes (Musitu y Cava, 2003; Ryan, Kalil y Leininger, 2009).

El apoyo social es una de las áreas de investigación más activas de las últimas décadas (v.g., Gracia y Herrero, 2004; Herrero y Gracia, 2007). Caplan (1974) destacó que el apoyo social podría funcionar como un protector ante la aparición de problemas; de tal forma que las personas que pertenecen a diversos grupos de apoyo, en diferentes contextos de la comunidad se pueden sentir inmunizadas ante el mundo estresante. El apoyo social se refiere a "la percepción o la experiencia de que uno es amado y cuidado, estimado y valorado, y es parte de una red social de asistencia mutua y obligaciones" (Taylor et al., 2004, p. 355, véase también Barrón, 1996; Gracia, 1997; Wills y Shinar, 2000). El apoyo social es un concepto claramente interactivo, es una transacción interpersonal de ayuda que se produce entre las fuentes de apoyo y el receptor de la ayuda, que puede implicar emociones, ayuda material e información y que se da en un contexto determinado (familiar, escolar, etc.). Generalmente se adopta una concepción multidimensional del mismo (por ejemplo, Cohen y Wills, 1985; Sarason, Levine, Bashman y Sarason, 1983). Según Laireiter y Baumann (1992) el apoyo social consta de cinco componentes: las redes de apoyo, el clima o ambiente de apoyo, el apoyo realizado, el apoyo percibido y el contexto donde se produce. También se distinguen diferentes dimensiones de apoyo social y tres han sido los tipos de apoyo que se han venido reflejando en la mayor parte de las clasificaciones: emocional, instrumental e informacional (Schaefer, Coyne y Lazarus, 1981). El apoyo emocional representa el sentimiento personal de ser amado y la seguridad de poder confiar en 
alguien. El apoyo instrumental, hace referencia a la posibilidad de poder disponer de ayuda directa y el informacional consiste en la provisión de consejo o guía para ayudar a las personas a resolver sus problemas. Cada tipo de apoyo responde a las diferentes funciones del apoyo social y se relaciona con unas necesidades específicas.

A pesar de este carácter multidimensional, generalmente los investigadores utilizan medidas que no hacen distinción entre las dimensiones de apoyo y las fuentes que lo proporcionan. Gran parte de los estudios obtienen sus resultados centrándose principalmente en el análisis del apoyo emocional, sin tener en cuenta otras funciones del apoyo como la instrumental e informacional, mientras que son muy escasos los estudios que incorporan otros tipos de apoyo (Malecki y Demaray, 2002). Sin embargo, los estudios han revelado diferencias entre los diferentes tipos de apoyo (por ejemplo, Clark-Lempers, Lempers y Ho, 1991) y los diferentes proveedores de apoyo (Procidiano y Heller, 1983). Así algunas investigaciones concluyen que los profesores en el contexto escolar principalmente muestran apoyo informacional (Malecki y Demaray, 2003) y que los padres prestan más apoyo instrumental que los amigos (Del Valle, Bravo y López, 2010).

Este aspecto lo consideramos muy importante ya que la distinción de los diferentes tipos de apoyo y de las fuentes que lo proporcionan en un contexto determinado nos permitirán conocer mejor de forma específica la experiencia real de apoyo. Este hecho es especialmente importante en la población adolescente ya que las relaciones que estos mantienen con su entorno son múltiples y entre ellas pueden mencionarse las que establece con su familia, con sus compañeros/as y con los profesores. Disponer de personas de confianza a las que poder expresar emociones, problemas o dificultades, escuchar su opinión, o simplemente tener la sensación de ser escuchados y aceptados como personas, ha demostrado tener un fuerte impacto en la capacidad de la persona para afrontar adecuadamente situaciones difíciles y estresantes (Lin y Ensel, 1989; Musitu, Buelga, Lila y Cava, 2001) y esto es especialmente importante para la población inmigrante (Martínez, García y Maya, 1999). Además estudios recientes han comprobado que la integración social y la percepción de apoyo se relacionan positivamente con el bienestar de la población inmigrante latina en nuestro país (Herrero, Fuente y Gracia, 2011).

Por ello es fundamental analizar el apoyo social desde la perspectiva de las distintas fuentes y los diferentes contextos, como indican los modelos ecológicos y sistémicos (Bronfenbrenner, 1979; Franco y Levitt, 1998; Levitt, 2005). La presente investigación se basó en un marco ecológico (Bronfenbrenner, 1995), que subraya la importancia del desarrollo de las relaciones sociales de los jóvenes a través de microsistemas claves tales como el hogar y la escuela.

Hasta la fecha, la mayoría de la investigación se ha centrado en la familia, preferentemente en los padres y en las relaciones con los amigos, y en general se acepta que estas relaciones tienen un impacto importante en el desarrollo de los mismos (Smetana, Campione-Barr y Metzger, 2006; Woolley, Kol y Bowen, 2009). Sin embargo, las teorías ecológicas también hacen hincapié en la importancia de la escuela como un contexto social importante. En el modelo ecológico de Bronfenbrenner (2005) la familia y la escuela se sitúan en el microsistema y ambos contextos son igualmente relevantes (Siegler, Deloache y Eisenberg, 2006). Los adolescentes pasan gran parte de su tiempo en la escuela en compañía de profesores y compañeros de clase, y ambos influyen en el desarrollo del adolescente (Eccles y Roeser, 2003). Además, diferentes investigaciones han mostrado un impacto diferenciado y singular de los padres, amigos, profesores y compañeros de clase sobre los problemas de comportamiento, problemas emocionales, ajuste académico así como en el desarrollo de conductas positivas (Cava, 2011; Cook, Herman, Phillips y Settersten , 2002; Demaray y Malecki, 2002; Garnefski y Diekstra, 1996; La Greca y López, 1998), lo que demuestra la importancia de analizar diferentes fuentes de apoyo en el contexto escolar. El apoyo dispensado por los padres a los adolescentes ha sido ampliamente estudiado pero son más escasos los estudios que se centran en el papel del profesorado y de los compañeros de clase como fuentes de apoyo.

La investigación disponible ha mostrado que en la transición de la escuela primaria a la secundaria se produce una disminución de la percepción del profesorado como fuente de apoyo (Furman y Buhrmester, 1992; Malecki y Demaray, 2002), mientras que el apoyo de los compañeros parece sufrir menos cambios (Harter, 1985). Sin embargo, el apoyo de los profesores es un aspecto clave en relación a los buenos resultados escolares de los estudiantes de minorías étnicas (Antrop-González, 2006; Johns, 2001). En cuanto a los compañeros, una visión global de las investigaciones realizadas en las últimas décadas en torno a las relaciones sociales de los jóvenes con sus iguales han demostrado que ser aceptado por los iguales se asocia positivamente a un buen ajuste escolar, menor probabilidad de fracaso académico, y a una mayor probabilidad de desarrollar relaciones sociales exitosas (Farrington, 1993; Slaby y Roedell, 1982). Existen suficientes datos que señalan que las relaciones con los iguales y la percepción de apoyo, facilitan el ajuste psicológico del adolescente. Así, unas buenas relaciones con los compañeros se han asociado con una alta autoestima (Robinson, 1995) y un menor riesgo de tener problemas emocionales y de conducta (Berndt y Savin-Williams, 1993; Coie y Dodge, 1998). La investigación sobre los jóvenes adolescentes inmigrantes y étnicamente diversos sugiere que, además los cambios significativos en las relaciones con las familias, los amigos y los profesores pueden hacer más difícil la transición a la escuela secundaria (Azmitia, Cooper y Brown, 2009; Phelan, Davidson y Cao, 1991; Seidman, Allen, Aber, Mitchell y Feinman, 1994).

A pesar del crecimiento de la población inmigrante y de que los estudios muestran que este grupo puede sentirse especialmente marginado (Turney y Kao, 2009), pocos estudios examinan la percepción y las diferencias de apoyo social 
entre las minorías y la población autóctona (v.g., Navas, García, Rojas, Pumares y Cuadrado, 2006; Oppedal, Roysamb y Lackland, 2004). Se sabe poco de las relaciones que establecen los jóvenes de diferentes grupos étnicos y culturales y pocos estudios incluyen más de un grupo étnico (Navas y Cuadrado, 2003; Phinney, Ong y Madden, 2000). Las diferencias étnicas son un importante predictor del tipo y cantidad de apoyo social del que se dispone ya que la variación cultural va unida a las diferencias en el apoyo social (Kim y McKenry 1998). Es importante conocer las múltiples interrelaciones que se crean entre las minorías y las sociedades de acogida dentro del ámbito educativo debido a que pueden llegar a provocar la existencia de conflictos. La experiencia de sufrir violencia diaria, o incluso, observarla, tienen consecuencias muy negativas para el desarrollo infantil y adolescente. Fernández et al. (2011) emplearon el constructo de violencia escolar cotidiana para distanciarlo del acoso entre iguales. Este se refiere a comportamientos de indisciplina, agresiones, insultos, exclusiones de intensidad leve o media y alta frecuencia entre los iguales. También se define como comportamientos intencionados para provocar daño, de carácter verbal y/o físico en respuesta a una provocación, utilizándose para ello la coacción, agresión o intimidación hacia los compañeros de clase (Trianes, 2000). Diversos estudios han puesto de manifiesto los efectos perniciosos de sufrir violencia escolar en el ámbito psicopatológico (Greve y Wilmers, 2003), afectivo-social (Bernstein y Watson, 1997; Pellegrini, Bartini y Brooks, 1999) y en el aprendizaje y rendimiento académico (Beale, 2001). Igualmente, sufrir violencia escolar de baja intensidad sin episodios de acoso o maltrato también puede predecir psicopatología e inadaptación personal (Cangas, Gázquez, Pérez- Fuentes, Padilla y Miras, 2007). Un ambiente escolar de violencia diaria provoca un mayor número de comportamientos agresivos entre el alumnado, lo cual se asocia al deterioro del clima social de clase, afectando de esta forma a todo el contexto educativo, e impidiendo el normal desarrollo de la docencia y de las actividades de clase (Sánchez, Rivas y Trianes, 2006; Westling, 2002). En cambio, un clima social positivo presenta efectos particularmente constructivos en estudiantes que pueden tener riesgos de dificultades académicas, emocionales o comportamentales (Felner et al., 1995). Actualmente, el concepto de clima social dentro del ámbito educativo se suele definir como la calidad de las interacciones interpersonales en el centro escolar (Emmons, Comer y Haynes, 1996), o también como la percepción, por parte de estudiantes y profesores, de bienestar personal, sentimientos positivos de sentirse aceptado y ser valioso para los demás en la convivencia diaria (Trianes, 2000). Algunas investigaciones actuales relacionan la mejora de las relaciones sociales en la clase, particularmente en estudiantes con riesgo, con la prevención de dificultades, asociándose a un desarrollo saludable, un aprendizaje óptimo y reductor de conductas desadaptativas (Ladd, 1990; Parker y Asher, 1987), concretamente en el caso de estudiantes varones (Kuperminc, Blatt y Leadbeater, 1997) y con un aumento de la satisfacción vital (Martínez, Moreno, Amador y Orford, 2011).

Por tanto, teniendo en cuenta los hallazgos de los estudios sobre el apoyo social, el clima social y los conflictos escolares pensamos que es necesario profundizar en relaciones entre estas variables en contextos escolares multiculturales. La coexistencia de estudiantes inmigrantes y autóctonos es una realidad dentro del sistema educativo actual, lo que ha abierto las puertas a los estudios que analizan el proceso de integración de los inmigrantes. En cambio, aunque las personas de etnia gitana han estado siempre presentes en nuestra sociedad, son escasos los estudios que analizan las relaciones de interacción del alumnado de etnia gitana con el resto del alumnado. En esta investigación queremos explorar una realidad presente en nuestras aulas, que es la existencia de relaciones interculturales. En concreto, este trabajo pretende conocer la percepción de apoyo social emocional, instrumental e informacional entre los alumnos autóctonos, inmigrantes y autóctonos de etnia gitana que conviven en el aula; analizar el apoyo social recibido por parte de las principales fuentes de apoyo en el aula (compañeros/as y profesorado) y comprobar si la percepción de apoyo social de los estudiantes se relaciona positivamente con el clima social del centro escolar y la disminución de conflictos en clase. En base a los estudios previos se espera que los estudiantes inmigrantes y de etnia gitana perciban menos apoyo social que el resto de los alumnos autóctonos y que la percepción del apoyo social de estudiantes y profesores se relacione positivamente con el clima social y la disminución de conflictos.

\section{Método}

\section{Participantes}

Participaron en este estudio 512 estudiantes pertenecientes a diferentes Centros Escolares Públicos de la Provincia de Málaga donde se congregan la mayor parte de las familias gitanas e inmigrantes de la zona. La selección de los participantes se realizó mediante un muestreo estratificado por conglomerados. Las unidades de muestreo fueron los centros educativos públicos donde se concentran estudiantes inmigrantes, autóctonos y de etnia gitana. Los estratos se establecieron en función de la variable curso $\left(1^{\circ}, 2^{\circ}, 3^{\circ}\right.$ y $4^{\circ}$ de la ESO).

Los participantes fueron 261 chicos $(51 \%)$ y 251 chicas $(49 \%)$ entre los 12 y 17 años, $(M=14.14, D T=1.30)$ de los cuáles un $9 \%$ del alumnado es inmigrante, un $4 \%$ es de etnia gitana y un $87 \%$ pertenece al resto del alumnado autóctono. Estos porcentajes son representativos de la realidad de los centros educativos situados en zonas donde se concentran población gitana e inmigrante ya que la población de los inmigrantes representa en torno a un 9\% (Ministerio de Educación, 2010) y los datos de la población gitana indican que sólo un $24 \%$ de estos alumnos sigue estudiando después de primaria, existiendo un elevado absentismo y un alto índice de abandono en la etapa de la E.S.O. ya que el $80 \%$ de los 
alumnos gitanos que comienzan la Educación Secundaria Obligatoria abandonan estos estudios antes de finalizar (Andrés, 2006).

Para el estudio se han diferenciado tres grupos: Alumnado inmigrante, cuyas familias se han desplazado de su país de origen a nuestro país para establecerse aquí en busca de un futuro mejor. Alumnado de etnia gitana, que han nacido en nuestro país y que son de etnia gitana. Alumnado autóctono, que han nacido en nuestro país y no pertenecen a ninguna de las dos categorías anteriores. En adelante utilizaremos esta denominación para referirnos a los tres grupos de estudiantes que han participado en el estudio.

Según la edad, el tamaño de la muestra quedó como sigue: 12 años $(N=55) ; 13$ años $(N=113) ; 14$ años $(N=149)$; 15 años $(N=115) ; 16$ años $(N=61) ; 17$ años $(N=19)$. Por otro lado, la muestra de alumnado inmigrante está representada por 25 nacionalidades diferentes que se han agrupado en 4 grupos geográficos principales: Latinoamérica (49.5\%), Europa del Este $(25.4 \%)$, África $(23.8 \%)$ y otros países de Europa (1.3\%). El tiempo medio de residencia de los estudiantes inmigrantes en nuestro país es de 13.3 años $(D T=$ 3.03). Según el curso al que pertenece el alumnado, el $29.5 \%$ estudia $1^{\circ}$ de E.S.O., el $28.7 \%$ estudia $2^{\circ}$ de E.S.O., el 27.1\% estudia $3^{\circ}$ de E.S.O. y el 14.6\% estudia $4^{\circ}$ de E.S.O.

\section{Instrumentos}

Características Sociodemográficas: Los datos personales de los participantes que se han recogido han sido: la edad, el sexo (hombre, mujer), el curso $\left(1^{\circ} \mathrm{ESO}, 2^{\circ} \mathrm{ESO}, 3^{\circ} \mathrm{ESO}, 4^{\circ}\right.$ ESO), el origen étnico (estudiante autóctono, estudiante de etnia gitana, estudiante inmigrante), el país de nacimiento, el de sus padres y, en su caso, el tiempo de residencia en España.

Percepción de Conflictos: Se elaboran para el estudio una serie de cuestiones en las que se les pregunta a los estudiantes por los problemas de convivencia y los conflictos más frecuentes en el aula.

Hay una pregunta referente a las preferencias de relación entre los compañeros, distinguiendo entre $1=$ compañeros/as inmigrantes, $2=$ de etnia gitana, $3=$ autóctonos y $4=$ todos por igual. Hay dos cuestiones relativas a la percepción de conflictos que se responden conforme a una escala tipo likert ( $1=$ Nunca, $5=$ Muchas veces $)$ y otra que evalúa los tipos de conflictos que suelen darse. Se indican 7 tipos de conflictos pudiendo señalar todas las opciones necesarias. Una puntuación alta indica una mayor percepción de conflictos:

1) ¿Con qué compañeros/as prefieres relacionarte? (1=Compañeros $/$ as inmigrantes, 2=Compañeros $/$ as de etnia gitana, $3=$ Compañeros $/$ as autóctonos, $4=$ Todos por igual), 2) ¿Existen problemas de convivencia en el aula? $(1=$ Nunca, $2=$ Muy pocas veces, $3=$ Algunas veces, $4=$ Bastantes veces, $5=$ Muchas veces), 3) ¿Suelen producirse conflictos por las diferencias culturales/étnicas entre compañeros/as? (1= Nunca, 2= Muy pocas veces, 3= Algunas veces, 4= Bastantes veces, $5=$ Muchas veces), 4) ¿Qué tipos de conflictos suelen darse? Señala todas las opciones que consideres $(1=$ insultos y burlas; $2=$ agresiones físicas; $3=$ robos; $4=$ intimidación y amenazas; $5=d e-$ terioro del material; $6=$ bumillaciones y descalificaciones; $7=$ acoso; $8=$ otros).

Cuestionario de Clima Social del Centro Escolar (CECSCE) de Trianes, Blanca, De la Morena, Infante y Raya (2006). Se utilizó para conocer la visión que tiene el alumnado del clima social existente en sus aulas y en el Centro Escolar. Está formado por 14 ítems con un formato de respuesta tipo Likert $(1=$ nunca, $5=$ muchas veces $)$. El CECSCE muestra una estructura factorial estable Oblimin con dos factores. Los 8 primeros ítems reflejan el clima social del centro escolar y se han agrupado en un primer factor identificado como Clima referente al Centro (v.g., Cuando los estudiantes rompen las reglas, son tratados justamente) y los últimos 6 ítems hacen referencia principalmente a la relación alumno-profesor, siendo este factor denominado Clima referente al Profesorado (v.g., $M e$ siento cómodo hablando con mis profesores de mis problemas). El instrumento muestra en nuestro estudio un alto índice de consistencia interna $(a=.85)$.

Cuestionario de Apoyo Social Recibido y Percibido (ASORPE) (Hombrados, García y Moscato, 2005), permite analizar el contexto donde se produce el apoyo, las fuentes de apoyo distinguiendo entre compañeros inmigrantes, de etnia gitana, compañeros autóctonos y profesorado y el tipo de apoyo recibido: emocional (v.g., Te ofrecen cariño, afecto o te escuchan cuando quieres hablar y expresar tus sentimientos); instrumental (v.g., Te harían un favor si lo necesitaras o están dispuestos a hacer cosas concretas por ti como ayudarte con las tareas de clase o en cualquier otra actividad) e informacional (v.g., Te dan consejos útiles e información para resolver dudas, problemas o las cosas que debes hacer a diario). Para cada fuente de apoyo y para cada tipo de apoyo se evalúa la frecuencia y la satisfacción del mismo. El cuestionario utiliza una escala de respuesta tipo Likert de 1 a 5 puntos, tanto para la frecuencia de apoyo recibido $(1=$ rara vez y $5=$ siempre), así como para medir el grado de satisfacción con el mismo apoyo (1= insatisfecho y $5=$ muy satisfecho). El instrumento muestra un alto índice de consistencia interna $(a=.90)$.

\section{Procedimiento}

Se contactó con la Dirección y el Departamento de Orientación de los Centros en los que se iban a aplicar los cuestionarios. Una vez que el equipo directivo aceptó la propuesta, se informó al profesorado y al Consejo Escolar de que el alumnado de la E.S.O. iba a cumplimentar unos cuestionarios en la hora de tutoría. El Departamento de Orientación elaboró un horario con las horas de tutoría de los cursos a los que se les iba a pasar los cuestionarios. El investigador se comprometió a facilitar los resultados de la investigación al Departamento de Orientación a modo de agradecimiento por su participación y colaboración. En la hora de tutoría el investigador repartía a cada estudiante los cuestionarios, daba las instrucciones para rellenarlos y les insistía en el anonimato y confidencialidad de la información 
aportada. Un colaborador estaba presente durante todo el proceso de cumplimentación de los instrumentos. En total se distribuyeron 521 cuestionarios y todos fueron entregados al entrevistador, se desestimaron 9 cuestionarios por estar incompletos. Se consiguió una tasa de respuesta del 100\%.

\section{Resultados}

Para analizar el apoyo que los estudiantes reciben del profesorado se ha empleado un ANOVA de medidas repetidas (Tabla 2) utilizando como variable intrasujeto el tipo de apoyo (emocional, instrumental e informacional) proporcionado tanto para la frecuencia como para la satisfacción con el apoyo (Tabla 1), en cada uno de los grupos de estudiantes (inmigrantes, etnia gitana y autóctonos). Los resultados de la Tabla 2 muestran que hay diferencias significativas en la percepción de apoyo social recibido del profesorado, tanto en las dimensiones de frecuencia como de satisfacción. Las medias de la Tabla 1 muestran que el apoyo informacional es el que más frecuentemente proporcionan los profesores/as y es también con el que más satisfecho se encuentran los estudiantes de los tres grupos analizados (inmigrantes, etnia gitana y autóctonos)

Tabla 1. Medias y desviaciones típicas de la frecuencia y satisfacción de los tipos de apoyo social recibido del profesorado en cada uno de los tres grupos de estudiantes.

\begin{tabular}{lllllll}
\hline Origen & Tipo de apoyo & \multicolumn{2}{l}{ Frecuencia } & \multicolumn{3}{l}{ Satisfacción } \\
\hline \multirow{3}{*}{ Inmigrante } & $M$ & $D . T$. & $N$ & $M$ & $D . T$. \\
& Emocional & 3.04 & 1.53 & 46 & 3.21 & 1.53 \\
& Instrumental & 2.30 & 1.57 & 46 & 2.69 & 1.67 \\
& Informacional & 3.89 & 1.33 & 46 & 3.80 & 1.42 \\
Etnia gitana & Emocional & 2.47 & 1.21 & 19 & 3.21 & 1.39 \\
& Instrumental & 1.68 & 1.00 & 19 & 2.36 & 1.57 \\
& Informacional & 3.52 & 1.50 & 19 & 3.94 & 1.17 \\
& Emocional & 3.03 & 1.34 & 447 & 3.39 & 1.43 \\
& Instrumental & 1.99 & 1.31 & 447 & 2.64 & 1.62 \\
& Informacional & 3.84 & 1.33 & 447 & 3.97 & 1.29 \\
\hline
\end{tabular}

Tabla 2. Análisis de varianza de medidas repetidas para Tipo de Apoyo Social proporcionado por el profesorado (Frecuencia y Satisfacción) en cada uno de los tres grupos de estudiantes.

\begin{tabular}{|c|c|c|c|c|c|c|}
\hline \multirow[t]{2}{*}{ Origen } & \multicolumn{2}{|l|}{ Fuente de variación } & \multicolumn{2}{|c|}{ Frecuencia } & \multicolumn{2}{|c|}{ Satisfacción } \\
\hline & & $g l$ & $F$ & $p$ & $F$ & $p$ \\
\hline Inmigrante & Tipo de apoyo social & 2 & 27.19 & .000 & 14.30 & .000 \\
\hline Etnia gitana & Tipo de apoyo social & 2 & 22.28 & .000 & 14.10 & .000 \\
\hline Autóctono & Tipo de apoyo social & 2 & 386.72 & .000 & 194.97 & .000 \\
\hline
\end{tabular}

Para comprobar si existen diferencias entre el apoyo percibido procedente del profesorado en los tres grupos considerados se utiliza la prueba de Kruskal-Wallis con el origen de los alumnos/as como variable independiente y el apoyo social como variable dependiente. La utilización de esta prueba no paramétrica se debe a la gran desigualdad en el tamaño de la muestra de los estudiantes y por lo tanto no se pueden garantizar los supuestos paramétricos.

Respecto a la frecuencia del apoyo emocional que prestan los profesores/as se encuentran diferencias significativas entre los estudiantes inmigrantes, de etnia gitana y autóc- tonos $\chi^{2}=3.20 p=.02$. Los estudiantes de etnia gitana son los que reciben menos frecuentemente el apoyo emocional del profesorado. Para la frecuencia del apoyo instrumental recibido del profesorado no se han encontrado diferencias significativas entre los tres grupos de estudiantes $\chi^{2}=1.89$ $p=.38$. Tampoco se han encontrado diferencias significativas para la frecuencia del apoyo informacional entre los estudiantes $\chi^{2}=1.02 p=.59$. Aunque son los estudiantes de etnia gitana los que perciben con menor frecuencia apoyo instrumental e informacional del profesorado. Respecto a la satisfacción con el apoyo recibido de los profesores/as no se encuentran diferencias significativas para el apoyo emocional entre los tres grupos de estudiantes $\chi^{2}=.81 p=.66$; ni para el apoyo instrumental $\chi^{2}=.46 p=.70$; ni tampoco para la satisfacción con el apoyo informacional $\chi^{2}=.30 p=.80$. Aunque en todos los casos las medias de satisfacción con el apoyo son más pequeñas para los estudiantes de etnia gitana.

Los resultados muestran que en general no hay diferencias significativas en cuanto a la frecuencia y satisfacción con el apoyo recibido del profesorado según el grupo al que pertenecen los estudiantes. Únicamente las diferencias han sido significativas para la frecuencia del apoyo emocional, siendo los alumnos/as de etnia gitana los que perciben con menor frecuencia este tipo de apoyo. Los estudiantes autóctonos y los inmigrantes son los que muestran medias más parecidas mientras que los estudiantes de etnia gitana son los que perciben menos apoyo del profesorado.

En cuanto al apoyo que los estudiantes reciben de sus compañeros/as de clase la Tabla 3 presenta los resultados de la frecuencia y de la satisfacción con el tipo de apoyo social recibido (emocional, instrumental e informacional) por parte de las tres fuentes de apoyo analizadas. En la Tabla 4 se muestra el análisis de varianza de medidas repetidas para la frecuencia y satisfacción del apoyo social, con la fuente y los tipos de apoyo social; ambas como variables intrasujeto, cada una de ellas con tres niveles. Todo ello se realiza por separado para cada uno de los tres tipos de alumnos estudiados.

Para el alumnado inmigrante la Tabla 4 indica que hay diferencias significativas tanto en la fuente que proporciona el apoyo como en el tipo de apoyo percibido. Las medias de la Tabla 3 muestran que el apoyo percibido procede principalmente de los compañeros/as autóctonos, seguido de los inmigrantes y por último de los compañeros/as de etnia gitana. Los estudiantes inmigrantes perciben que el apoyo emocional e informacional procede más frecuentemente de los compañeros autóctonos y están también más satisfechos con este apoyo. En cambio, el apoyo instrumental, que está más relacionado con hacer cosas concretas, proviene más de los estudiantes inmigrantes. En todos los casos tanto para la frecuencia como para la satisfacción los estudiantes de etnia gitana son los que prestan menos apoyo a los compañeros /as inmigrantes. En cambio, los estudiantes autóctonos son los que prestan más apoyo emocional e informacional tanto en las dimensiones de frecuencia como de satisfacción. Res- 
pecto al tipo de apoyo, es el apoyo emocional el más importante para los estudiantes inmigrantes.

Tabla 3. Medias (M), desviaciones típicas (D.T.) y número de personas $(\mathrm{N})$ de la Fuente de Apoyo Social y el Tipo de Apoyo Social (Frecuencia y Satisfacción) en cada uno de los tres grupos de estudiantes

\begin{tabular}{|c|c|c|c|c|c|c|c|}
\hline \multirow[t]{2}{*}{ Origen } & \multirow[t]{2}{*}{$\begin{array}{l}\text { Fuente de } \\
\text { aроyo }\end{array}$} & \multirow[t]{2}{*}{$\begin{array}{l}\text { Tipo de } \\
\text { ароуо }\end{array}$} & \multicolumn{3}{|c|}{ Frecuencia } & \multicolumn{2}{|c|}{ Satisfacción } \\
\hline & & & $\bar{M}$ & D.T. & $N$ & $M$ & D.T. \\
\hline \multirow[t]{9}{*}{ Inmigrante } & Inmigrantes & Emocional & 3.32 & 1.44 & 46 & 3.45 & 1.50 \\
\hline & & Instrumental & 3.02 & 1.49 & 46 & 3.36 & 1.48 \\
\hline & & Informacional & 3.28 & 1.50 & 46 & 3.41 & 1.45 \\
\hline & Etnia gitana & Emocional & 2.04 & 1.33 & 46 & 2.15 & 1.42 \\
\hline & & Instrumental & 1.71 & 1.08 & 46 & 1.67 & 1.03 \\
\hline & & Informacional & 1.95 & 1.33 & 46 & 2.06 & 1.48 \\
\hline & Autóctonos & Emocional & 3.60 & 1.34 & 46 & 3.82 & 1.32 \\
\hline & & Instrumental & 2.89 & 1.41 & 46 & 3.26 & 1.59 \\
\hline & & Informacional & 3.60 & 1.40 & 46 & 3.78 & 1.39 \\
\hline \multirow[t]{9}{*}{$\begin{array}{l}\text { Etnia } \\
\text { gitana }\end{array}$} & Inmigrantes & Emocional & 2.21 & 1.27 & 19 & 2.94 & 1.64 \\
\hline & & Instrumental & 2.36 & 1.21 & 19 & 3.15 & 1.57 \\
\hline & & Informacional & 2.47 & 1.30 & 19 & 2.84 & 1.34 \\
\hline & Etnia gitana & Emocional & 3.57 & 1.34 & 19 & 3.73 & 1.32 \\
\hline & & Instrumental & 3.31 & 1.49 & 19 & 3.84 & 1.25 \\
\hline & & Informacional & 3.31 & 1.24 & 19 & 3.94 & .97 \\
\hline & Autóctonos & Emocional & 3.52 & 1.01 & 19 & 3.68 & 1.20 \\
\hline & & Instrumental & 2.89 & 1.04 & 19 & 3.31 & 1.20 \\
\hline & & Informacional & 3.47 & 1.07 & 19 & 3.78 & 1.22 \\
\hline \multirow[t]{9}{*}{ Autóctono } & Inmigrantes & Emocional & 3.10 & 1.41 & 447 & 3.37 & 1.46 \\
\hline & & Instrumental & 2.38 & 1.43 & 447 & 2.87 & 1.55 \\
\hline & & Informacional & 2.85 & 1.46 & 447 & 3.18 & 1.49 \\
\hline & Etnia gitana & Emocional & 2.38 & 1.54 & 447 & 2.66 & 1.64 \\
\hline & & Instrumental & 1.93 & 1.39 & 447 & 2.38 & 1.61 \\
\hline & & Informacional & 2.19 & 1.50 & 447 & 2.49 & 1.62 \\
\hline & Autóctonos & Emocional & 4.05 & 1.12 & 447 & 4.20 & 1.10 \\
\hline & & Instrumental & 3.50 & 1.31 & 447 & 3.87 & 1.28 \\
\hline & & Informacional & 3.96 & 1.13 & 447 & 4.13 & 1.11 \\
\hline
\end{tabular}

Tabla 4. Análisis de varianza de medidas repetidas para Fuente y Tipo de Apoyo Social (Frecuencia y Satisfacción) en cada uno de los tres grupos de estudiantes

\begin{tabular}{lllllll}
\hline Origen & Fuente de Variación & \multicolumn{3}{c}{ Frecuencia } & \multicolumn{2}{c}{ Satisfacción } \\
\cline { 3 - 7 } & & $g l$ & $F$ & $p$ & $F$ & $p$ \\
\hline \multirow{2}{*}{ Inmigrante } & Fuente del apoyo & 2 & 29.73 & .000 & 34.48 & .000 \\
& social & & & & & \\
& Tipo de apoyo social & 2 & 8.31 & .000 & 5.68 & .005 \\
& Fuente X Tipo & 4 & 1.89 & .114 & 1.83 & .125 \\
\hline Etnia & Fuente del apoyo social & 2 & 7.64 & .002 & 4.94 & .003 \\
gitana & Tipo de apoyo social & 2 & 1.29 & .288 & .18 & .834 \\
& Fuente X Tipo & 4 & 1.06 & .380 & 1.19 & .319 \\
\hline \multirow{2}{*}{ Autóctono } & Fuente del apoyo social & 2 & 313.45 & .000 & 228.16 & .000 \\
& Tipo de apoyo social & 2 & 131.35 & .000 & 65.27 & .000 \\
& Fuente X Tipo & 4 & 5.45 & .000 & 3.98 & .003 \\
\hline
\end{tabular}

Respecto al alumnado de etnia gitana, hay diferencias significativas en cuanto a la fuente que proporciona el apoyo (ver Tabla 4). En la Tabla 3 se observa que los estudiantes de etnia gitana son los que más frecuentemente proporcionan los tres tipos de apoyo a sus compañeros/as de etnia gitana. También se encuentran más satisfechos con el apoyo emocional, instrumental e informacional que estos le pro- porcionan, ya que la frecuencia y la satisfacción con los tres tipos de apoyo que reciben de los demás compañeros/as inmigrantes y autóctonos es significativamente menor. Puede afirmarse que la fuente principal de apoyo en el contexto del aula para los estudiantes de etnia gitana son sus compañeros/as también de etnia gitana, porque les prestan apoyo más frecuentemente y también están más satisfechos con el apoyo que éstos les proporcionan. Los estudiantes de los que perciben menos apoyo son los alumnos/as inmigrantes.

En el caso de los estudiantes autóctonos se han hallado efectos principales y de interacción de las dos variables ( $\mathrm{Ta}$ bla 4). Los estudiantes autóctonos perciben que el apoyo emocional, instrumental e informacional procede más frecuentemente de los compañeros autóctonos y están también más satisfechos con el apoyo que estos les proporcionan (ver Tabla 3). Los estudiantes de los que perciben menos apoyo son los alumnos/as de etnia gitana. En cuanto a los tipos de apoyo, el apoyo emocional es el que más valoran. También se produce para este grupo de estudiantes una interacción entre el tipo de apoyo y la fuente que lo proporciona ya que los datos indican que el apoyo emocional que le proporcionan los compañeros autóctonos es significativamente el más importante.

Para comprobar si los tres grupos de alumnos/as estudiados perciben diferencias en cuanto al apoyo que le proporcionan sus compañeros/as (inmigrantes, de etnia gitana y autóctonos) se utiliza la prueba de Kruskal-Wallis con el origen de los alumnos/as como variable independiente y como variables dependientes la frecuencia y satisfacción con los tres tipos de apoyo social proporcionado por las tres fuentes. Posteriormente se procede a realizar la prueba $U$ de Mann Whitney entre los pares de grupos (ver Tabla 5). La utilización de estas pruebas no paramétricas se debe a la gran desigualdad en el tamaño de la muestra de los estudiantes.

Puede observarse en la Tabla 5 que las diferencias entre los 3 grupos son estadísticamente significativas $(\mathrm{p}<.05)$ salvo para las variables dependientes de la frecuencia del apoyo informacional proporcionado por los inmigrantes y para la satisfacción del apoyo informacional proporcionado por los autóctonos.

La comparación del apoyo proporcionado por los inmigrantes entre los tres grupos ha arrojado los siguientes resultados (Ver Tablas 3 y 5):

Los estudiantes inmigrantes perciben más frecuentemente y están más satisfechos con el apoyo emocional e instrumental de los compañeros inmigrantes que los estudiantes de etnia gitana. También reciben más frecuentemente apoyo instrumental de su mismo grupo que los autóctonos, aunque no hay diferencias significativas para la frecuencia del apoyo emocional e informacional que reciben de los inmigrantes entre el grupo de inmigrantes y autóctonos ya que como se puede observar en la Tabla 3 las medias son parecidas. Tampoco se han encontrado diferencias significativas entre el grupo de inmigrantes y autóctonos en la satisfacción con los tres tipos de apoyo que proporcionan los estudiantes inmigrantes. 
La comparación del apoyo proporcionado por los estudiantes de etnia gitana entre los tres grupos ha arrojado los siguientes resultados (Ver Tablas 3 y 5).

Los estudiantes de etnia gitana reciben significativamente más apoyo emocional e instrumental de sus compañeros/as de etnia gitana que los estudiantes autóctonos e inmigrantes. Respecto a la satisfacción con el apoyo, el grupo de etnia gitana está significativamente más satisfecho con el apoyo emocional, instrumental e informacional que le proporciona el grupo de su misma etnia que los estudiantes inmigrantes y autóctonos.

La comparación del apoyo proporcionado por los estudiantes autóctonos entre los tres grupos ha arrojado los siguientes resultados (Ver Tablas 3 y 5).

Los estudiantes autóctonos reciben más frecuentemente apoyo emocional, instrumental e informacional de los compañeros inmigrantes que los estudiantes de etnia gitana, perciben significativamente más apoyo informacional de los compañeros/as de etnia gitana que los otros dos grupos y más apoyo emocional de los compañeros de etnia gitana que los inmigrantes. También perciben más frecuentemente apoyo emocional e instrumental de los autóctonos que los inmigrantes. Para el grupo de autóctonos y de etnia gitana se ha encontrado que los estudiantes autóctonos perciben más frecuentemente apoyo emocional, instrumental e informacional de los autóctonos que el grupo de etnia gitana y están significativamente más satisfechos con el apoyo emocional e instrumental que le proporcionan el grupo de autóctonos que el de etnia gitana. También hay diferencias significativas entre el grupo de inmigrantes y autóctonos ya que los autóctonos están más satisfechos con el apoyo emocional e instrumental que reciben de los compañeros/as de su mismo grupo.

La comparación entre grupos confirma que los estudiantes autóctonos perciben de los tres grupos significativamente más apoyo social que los estudiantes inmigrantes y de etnia gitana. Las mayores diferencias se producen entre el grupo de etnia gitana y el grupo de autóctonos.

Tabla 5. Prueba de Kruskal-Wallis (índice $\chi^{2}$ ) para la diferencia de medias entre los tres grupos de estudiantes (inmigrantes, de etnia gitana y autóctonos) y prueba U de Mann Whitney (índice U) para las comparaciones entre los pares de grupos. Se realizan todas las comparaciones para las 18 variables dependientes (Frecuencia y satisfacción del apoyo emocional, instrumental e informacional proporcionado por los estudiantes inmigrantes, de etnia gitana y autóctonos). $1=$ Grupo de inmigrantes. 2= Grupo de etnia gitana. 3= Grupo de autóctonos.

\begin{tabular}{|c|c|c|c|c|c|c|c|c|c|c|}
\hline \multirow{3}{*}{\multicolumn{2}{|c|}{ Variables dependientes }} & & \multicolumn{8}{|c|}{ Comparación entre los tres grupos de alumnos/as } \\
\hline & & & \multicolumn{2}{|c|}{$1-2-3$} & \multicolumn{2}{|c|}{$1-2$} & \multicolumn{2}{|c|}{$1-3$} & \multicolumn{2}{|c|}{$2-3$} \\
\hline & & & $\chi^{2}$ & $p$ & $U$ & $p$ & $U$ & $p$ & $U$ & $p$ \\
\hline & Inmigrantes & Emocional & 8.50 & .014 & 253.50 & .007 & 9310.0 & .278 & 2761.0 & .008 \\
\hline & & Instrumental & 7.72 & .021 & 323.50 & .094 & 7826.5 & .006 & 4201.0 & .935 \\
\hline & & Informacional & 5.01 & .082 & 299.00 & .042 & 8606.5 & .063 & 3613.0 & .260 \\
\hline \multirow[t]{8}{*}{ Frecuencia } & Etnia gitana & Emocional & 12.39 & .002 & 194.50 & .001 & 9100.5 & .185 & 2490.5 & .002 \\
\hline & & Instrumental & 16.52 & .000 & 177.50 & .001 & 9850.0 & .614 & 2122.5 & .001 \\
\hline & Autóctonos & Emocional & 9.85 & .007 & 405.00 & .638 & 8329.5 & .024 & 2984.5 & .019 \\
\hline & & Instrumental & 11.88 & .003 & 434.00 & .965 & 7770.0 & .005 & 3026.5 & .029 \\
\hline & & Informacional & 6.55 & .038 & 386.50 & .452 & 8988.5 & .139 & 3047.0 & .028 \\
\hline & Inmigrantes & Emocional & 11.32 & .051 & 5.325 & .041 & 9881.5 & .655 & 3661.5 & .296 \\
\hline & & Instrumental & 14.61 & .010 & 6.415 & .030 & 8446.0 & .041 & 3802.0 & .428 \\
\hline & & Informacional & 12.28 & .031 & .8242 & .010 & 9421.5 & .339 & 3629.0 & .272 \\
\hline \multirow[t]{5}{*}{ Satisfacción } & Etnia gitana & Emocional & 12.45 & .002 & 191.00 & .001 & 8477.5 & .044 & 2708.0 & .006 \\
\hline & & Informacional & 18.47 & .000 & 146.00 & .001 & 8759.5 & .087 & 2121.5 & .001 \\
\hline & Autóctonos & Emocional & 8.88 & .012 & 392.50 & .502 & 8502.0 & .033 & 3081.0 & .025 \\
\hline & & Instrumental & 10.52 & .005 & 428.00 & .894 & 8043.0 & .010 & 3068.0 & .031 \\
\hline & & Informacional & 4.44 & .108 & 414.50 & .734 & 8943.0 & .115 & 3447.5 & .132 \\
\hline
\end{tabular}

A continuación se presentan los resultados correspondientes a la percepción de conflictos en el aula y del clima social. Respecto a las preferencias en las relaciones con los compañeros y compañeras, hemos encontrado que el $30 \%$ prefiere relacionarse con todos por igual y el $66.3 \%$ prefiere relacionarse con los estudiantes autóctonos. Únicamente el $1.5 \%$ prefiere relacionarse con los estudiantes de etnia gitana y sólo el $2.3 \%$ prefiere a los estudiantes inmigrantes.

En cuanto al tipo de conflicto que habitualmente se produce entre el alumnado encontramos los siguientes resultados: La existencia de insultos y burlas entre compañeros/as se da en un porcentaje muy elevado (47\%), le siguen conductas de humillación y descalificaciones $(16 \%)$, intimida- ción y amenazas $(15 \%)$, deterioro del material $(9 \%)$, robos $(6 \%)$, agresiones físicas $(5 \%)$, acoso $(1 \%)$ y otros $(1 \%)$.

Para analizar la percepción de conflictos en el aula de los estudiantes, según la categoría a la que pertenecen, se utiliza la prueba de Kruskal-Wallis con el origen de los estudiantes como variable independiente y como variables dependientes los problemas de convivencia en el aula y la percepción de conflictos por las diferencias culturales/ étnicas. Esta misma prueba se utiliza para analizar la percepción de los estudiantes del clima social del centro escolar, siendo las variables dependientes las dimensiones de clima social en relación al centro y clima social en relación a los profesores. 
Encontramos que no hay diferencias significativas en la percepción de problemas de convivencia entre los estudiantes de etnia gitana $(M=3.00)$, inmigrantes $(M=2.59)$ y autóctonos $(M=2.64) \chi^{2}=2.90 p=.23$. Tampoco hay diferencias en la percepción de conflictos étnico/culturales entre los estudiantes de etnia gitana $(M=2.16)$, estudiantes inmigrantes $(M=2.04)$ y autóctonos $(M=1.81) \chi^{2}=4.17 p=.12$. $\mathrm{El}$ análisis del clima social no ha desvelado diferencias significativas atendiendo al tipo de alumnado. No hay diferencias en la percepción del clima social en relación al centro entre los estudiantes de etnia gitana $(M=2.96)$, inmigrantes $(M=$ $3.20)$ y autóctonos $(M=3.28) \chi^{2}=2.76 p=.25$, y no se encuentran diferencias significativas para la percepción del clima social en relación al profesorado entre los estudiantes de etnia gitana $(M=3.31)$, inmigrantes $(M=3.57)$ y autóctonos $(M=3.62) \chi^{2}=1.83 p=.40$. Sin embargo, hay que destacar que los estudiantes de etnia gitana son los que manifiestan las medias más bajas en la percepción del clima social y los que perciben más conflictos debidos a las diferencias culturales y más problemas de convivencia.

En el siguiente apartado se describen las relaciones existentes entre el apoyo social que reciben los estudiantes y la percepción de conflictos y el clima social del aula. La Tabla 6 presenta las correlaciones de Pearson del apoyo social de los estudiantes con la percepción del clima social del centro escolar, la percepción de problemas de convivencia en el aula y la percepción de conflictos étnico/culturales. Para el apoyo social de los estudiantes se han analizado las dimensiones de frecuencia y satisfacción con los tres tipos de apoyo: emocional, instrumental e informacional. Para el clima social se han analizado las dimensiones de clima de centro y clima de profesorado.

Tabla 6. Correlaciones del apoyo social de los estudiantes con el clima social del centro escolar, los problemas de convivencia y la percepción de conflictos interculturales.

\begin{tabular}{|c|c|c|c|c|}
\hline & $\begin{array}{l}\text { Clima } \\
\text { Centro }\end{array}$ & $\begin{array}{l}\text { Clima } \\
\text { Profesores }\end{array}$ & $\begin{array}{l}\text { Problemas de } \\
\text { convivencia }\end{array}$ & $\begin{array}{l}\text { Conflictos } \\
\text { étnico/culturales }\end{array}$ \\
\hline Frecuencia del apoyo emocional de los estudiantes inmigrantes & $.160(* *)$ & $.106(*)$ & $-.145(* *)$ & $-.130(* *)$ \\
\hline Frecuencia del apoyo instrumental de los estudiantes inmigrantes & $.103(*)$ & .051 &.$-123(*)$ & -.074 \\
\hline Frecuencia del apoyo informacional de los estudiantes inmigrantes & $.097(*)$ & .052 &.$-107(*)$ & $-.115(* *)$ \\
\hline Satisfacción con el apoyo emocional de los estudiantes inmigrantes & $.167(* *)$ & $.096(*)$ & $-.128(* *)$ & $-.126(* *)$ \\
\hline Satisfacción con el apoyo instrumental de los estudiantes inmigrantes & $.150(* *)$ & .079 & $-.150(* *)$ & $-.122(* *)$ \\
\hline Satisfacción con el apoyo informacional de los estudiantes inmigrantes & $.150(* *)$ & $.128(* *)$ & $-.096(*)$ & $-.130(* *)$ \\
\hline Frecuencia del apoyo emocional de los estudiantes de etnia gitana & .034 & .030 & $-.103(*)$ & .045 \\
\hline Frecuencia del apoyo instrumental de los estudiantes de etnia gitana & .005 & .012 & -.068 & .038 \\
\hline Frecuencia del apoyo informacional de los estudiantes de etnia gitana & .009 & .038 & $-.095(*)$ & .017 \\
\hline Satisfacción con el apoyo emocional de los estudiantes de etnia gitana & .078 & .062 & -.086 & .031 \\
\hline Satisfacción con el apoyo instrumental de los estudiantes de etnia gitana & .014 & .053 & -.086 & .038 \\
\hline Satisfacción con el apoyo informacional de los estudiantes de etnia gitana & .004 & .000 & -.076 & .035 \\
\hline Frecuencia del apoyo emocional de los estudiantes autóctonos & $.351(* *)$ & $.218(* *)$ & $-.153(* *)$ & $-.214(* *)$ \\
\hline Frecuencia del apoyo instrumental de los estudiantes autóctonos & $.170(* *)$ & $.190(* *)$ & -.073 & $-.140(* *)$ \\
\hline Frecuencia del apoyo informacional de los estudiantes autóctonos & $.256(* *)$ & $.182(* *)$ & $-.146(* *)$ & $-.111(*)$ \\
\hline Satisfacción con el apoyo emocional de los estudiantes autóctonos & $.349(* *)$ & $.278(* *)$ & $-.132(* *)$ & $-.216(* *)$ \\
\hline Satisfacción con el apoyo instrumental de los estudiantes autóctonos & $.207(* *)$ & $.239(* *)$ & $-.091(*)$ & $-.137(* *)$ \\
\hline Satisfacción con el apoyo informacional de los estudiantes autóctonos & $.259(* *)$ & $.212(* *)$ & $-.120(* *)$ & $-.139(* *)$ \\
\hline
\end{tabular}

** La correlación es significativa al nivel .01 (bilateral).* La correlación es significante al nivel .05 (bilateral)

Respecto a la percepción del apoyo que prestan los estudiantes inmigrantes los resultados muestran que la percepción del apoyo social emocional, informacional e instrumental de los estudiantes inmigrantes correlacionan positiva $y$ significativamente con la percepción positiva del centro escolar, tanto para la dimensión de frecuencia como de satisfacción con el apoyo social. En cambio, el clima referente al profesorado únicamente correlaciona positiva y significativamente con la frecuencia y satisfacción del apoyo social emocional y con la satisfacción del apoyo informacional que proporcionan estos estudiantes. La existencia de problemas de convivencia en el aula correlaciona negativamente y de forma significativa con todos los tipos de apoyo social analizados y tanto para la frecuencia como para la satisfacción del apoyo de los estudiantes inmigrantes. La percepción de apoyo social de los estudiantes inmigrantes también correlaciona significativamente y de forma negativa con la aparición de conflictos debidos a las diferencias culturales/étnicas para todos los tipos de apoyo y para las dimensiones de frecuencia y satisfacción, excepto en el caso de la frecuencia con que prestan apoyo instrumental los estudiantes inmigrantes que aun siendo una relación negativa no ha sido estadísticamente significativa.

En general, el apoyo social de los alumnos inmigrantes se relaciona más positivamente con la percepción del clima social referente al centro que con el clima social referente al profesorado. También el apoyo de los alumnos inmigrantes correlaciona negativamente con la existencia de conflictos étnico/culturales y con los problemas de convivencia en el aula.

En cuanto a la percepción del apoyo que prestan los estudiantes de etnia gitana los resultados indican que las correlaciones entre el apoyo que brindan estos estudiantes y el clima social del centro y de los profesores no han sido signi- 
ficativas. Tampoco han sido significativas las correlaciones con los problemas de convivencia en aula y los conflictos debidos a las diferencias culturales. Únicamente las correlaciones han sido estadísticamente significativas para la frecuencia del apoyo emocional y para la frecuencia del apoyo informacional ya que en la medida en que aumentan estos dos tipos de apoyo disminuyen los problemas de convivencia en el aula.

Los resultados sobre la percepción del apoyo que dan los estudiantes autóctonos indican que la frecuencia y satisfacción con los tres tipos de apoyo correlacionan positiva y significativamente con el aumento del clima social del centro y con el aumento de un clima social positivo en relación al profesorado. En cambio, la relación es negativa con los problemas de convivencia para todas las dimensiones del apoyo social analizadas y para los tres tipos de apoyo, excepto en el caso de la frecuencia con que prestan apoyo instrumental los estudiantes autóctonos, que aun siendo una relación negativa no ha sido estadísticamente significativa. Respecto a la existencia de conflictos producidos por las diferencias culturales/étnicas, los resultados también indican que las correlaciones son negativas y estadísticamente significativas para los tres tipos de apoyo que brindan estos estudiantes y tanto para las dimensiones de frecuencia como de satisfacción.

En general, el aumento de la frecuencia y satisfacción con el apoyo social que proporcionan los alumnos autóctonos se relaciona positivamente con el aumento del clima so- cial para las dos dimensiones: clima social del centro y clima social en relación al profesorado. En cambio, la frecuencia y satisfacción con los tres tipos de apoyo se relacionan negativamente con los problemas de convivencia en el aula y los conflictos debidos a las diferencias étnicas/culturales.

Los resultados de las correlaciones de Pearson del apoyo social del profesorado con el clima social del centro escolar y la percepción de conflictos se muestran en la Tabla 7. En ella se observa que tanto la frecuencia como la satisfacción para los tres tipos de apoyo (emocional, instrumental e informacional) correlacionan positiva y significativamente con las dos dimensiones del clima social del centro escolar analizadas: clima social del centro y clima social de profesores. Para los problemas de convivencia las correlaciones han mostrado que el apoyo proporcionado por el profesorado (emocional, instrumental e informacional) en las dos dimensiones de frecuencia y satisfacción se relacionan negativamente y de forma significativa. Es decir, los problemas de convivencia en el aula disminuyen conforme aumenta la percepción de apoyo del profesorado. Sin embargo, la relación del apoyo social del profesorado con la disminución de los conflictos producidos por las diferencias étnicas/culturales no ha sido significativa. Excepto para la satisfacción con el apoyo social emocional que proporciona el profesorado. Siendo ésta la única dimensión del apoyo social prestado por el profesorado que se relaciona con la disminución de los conflictos producidos por las diferencias étnicas y culturales.

Tabla 7. Correlaciones del apoyo social del profesorado con el clima social del centro escolar, los problemas de convivencia y la percepción de conflictos étnico/culturales.

\begin{tabular}{|c|c|c|c|c|}
\hline & $\begin{array}{l}\text { Clima } \\
\text { Centro }\end{array}$ & $\begin{array}{l}\text { Clima } \\
\text { Profesores }\end{array}$ & $\begin{array}{l}\text { Problemas } \\
\text { convivencia }\end{array}$ & $\begin{array}{l}\text { Conflictos } \\
\text { interculturales }\end{array}$ \\
\hline Frecuencia del apoyo emocional del profesorado & $.341(* *)$ & $.541(* *)$ & $-.145(* *)$ & -.066 \\
\hline Frecuencia del apoyo instrumental del profesorado & $.144(* *)$ & $.237(* *)$ & $-.157(* *)$ & .050 \\
\hline Frecuencia del apoyo informacional del profesorado & $316(* *)$ & $.471(* *)$ & $-.112(*)$ & -.053 \\
\hline Satisfacción con el apoyo emocional del profesorado & $355(* *)$ & $.511(* *)$ & $-.157(* *)$ & $-.090(*)$ \\
\hline Satisfacción con el apoyo instrumental del profesorado & $.149(* *)$ & $.253(* *)$ & $-.112(*)$ & .005 \\
\hline Satisfacción con el apoyo informacional del profesorado & $.301(* *)$ & $.457(* *)$ & $-.121(* *)$ & -.072 \\
\hline
\end{tabular}

\section{Discusión}

Los resultados de este estudio muestran la complejidad del papel que juega el apoyo social en un contexto escolar multicultural. La percepción de apoyo social varía en función al grupo étnico y cultural al que se pertenezca y también el apoyo percibido difiere según la fuente que lo proporciona.

Respecto al profesorado los datos indican que el apoyo informacional es el más valorado por los estudiantes, seguido del apoyo emocional y por último del apoyo instrumental, tanto en las dimensiones de frecuencia como de satisfacción, aunque es la satisfacción con el apoyo informacional el aspecto más valorado. En general, los estudiantes perciben que los profesores ofrecen consejos e información útiles para resolver dudas, problemas o las cosas que deben hacer a diario y están satisfechos con el apoyo que le dispensan. Aunque normalmente los estudios de apoyo social muestran que el apoyo social emocional es el más valorado y el que tiene repercusiones más beneficiosas para los individuos, hay que tener en cuenta que la satisfacción con el apoyo viene determinada, en gran parte, por las necesidades de los sujetos (Lin,1986). Este aspecto queda recogido en la Teoría de la Especificidad de Cohen y McKay (1984) quienes plantean que el apoyo social será más efectivo cuanto más se ajuste al problema que hay que dar respuesta. En el contexto escolar, para los estudiantes, tiene un gran valor el apoyo informacional que reciben de sus profesores.

Respecto a los estudiantes, los resultados muestran que hay diferencias significativas en cuanto al apoyo proporcionado por los estudiantes según los grupos analizados. Los estudiantes que perciben menos apoyo social de sus compañeros/as de clase son los de etnia gitana, a continuación le siguen los estudiantes inmigrantes y finalmente, los estudiantes autóctonos son los que perciben más apoyo social en el 
aula. Estos resultados confirman nuestra hipótesis en la que esperábamos encontrar que la percepción de apoyo de los estudiantes inmigrantes y de etnia gitana sería menor que la del resto de los alumnos autóctonos. También el apoyo social varía según la fuente que lo proporciona. Los estudiantes de etnia gitana perciben que el apoyo procede principalmente de su grupo de iguales de etnia gitana, al igual que ocurre con los estudiantes autóctonos que perciben que el apoyo procede principalmente de los otros estudiantes autóctonos. No ocurre así con el alumnado inmigrante que percibe apoyo tanto de sus iguales autóctonos como de los iguales inmigrantes. Estos resultados concuerdan con algunos estudios que sugieren una variación sustancial en la percepción de apoyo por el origen étnico, siendo las personas de grupos étnicos minoritarios las que tienen menos percepción de apoyo (Hao, 2003). Además otros estudios muestran que los inmigrantes pueden tener acceso a diferentes fuentes de apoyo, pero, en general, experimentan una desventaja significativa, ya que los inmigrantes y las minorías étnicas son más propensos a tener el apoyo de otras personas pertenecientes a su mismo grupo étnico, con escasos recursos, como ha ocurrido en nuestro estudio con la población gitana ( Kim y McKenry, 1998). En cambio, las relaciones positivas con la población autóctona incrementan la percepción de apoyo y la satisfacción vital (Dominguez y Hombrados, 2008). También se ha constatado que la presencia de autóctonos en las redes sociales de los inmigrantes y la satisfacción con el contacto mantenido con los miembros de la sociedad receptora facilitan el ajuste psicosocial de este grupo de población (Searle y Ward, 1990). En nuestra investigación los estudiantes inmigrantes mantienen relaciones con los compañeros autóctonos y están satisfechos con el apoyo que éstos le proporcionan y en general, perciben más apoyo que los estudiantes de etnia gitana que consideran que el apoyo procede principalmente de sus iguales también de etnia gitana. Además los resultados han mostrado que las minorías étnicas (población gitana e inmigrante) mantienen escaso contacto entre sí. Los inmigrantes prefieren relacionarse con la población autóctona e inmigrante, de la que perciben la mayor parte del apoyo. La población de etnia gitana establece fuertes lazos de interacción con los miembros de su mismo grupo étnico y este se convierte en su principal fuente de apoyo, aunque el limitarse a esta red social le hace percibir menos apoyo que el resto de compañeros inmigrantes y autóctonos.

En este trabajo queda reflejada la función de reciprocidad del apoyo social, cuando los recursos de la red son intercambiados de forma equitativa entre las partes es más probable que aumente la percepción de apoyo social (Hall y Wellman, 1985). Los resultados muestran que el intercambio de apoyo para los estudiantes de etnia gitana se produce dentro de su mismo grupo étnico con una escasa red social y son estos estudiantes los que perciben menos apoyo del resto de compañeros autóctonos e inmigrantes. También autóctonos e inmigrantes perciben un escaso apoyo social de los compañeros/as de etnia gitana. Sería necesario fomentar las redes sociales entre los diversos grupos para facilitar el intercambio de apoyo y que este se produjera de forma reciproca entre los diferentes grupos étnicos y culturales que conviven en el aula.

El profesorado y los compañeros/as han mostrado ser importantes fuentes de apoyo aunque el tipo de apoyo que se percibe de cada fuente ha sido diferente. Para el profesorado ha sido el apoyo informacional el más importante. Para los estudiantes hemos encontrado que los tres tipos de apoyo son importantes, aunque el apoyo social emocional, es el tipo de apoyo que proporcionan más frecuentemente y con el que más satisfechos se encuentran. Estos datos concuerdan con la mayoría de los estudios de apoyo social que muestran diferencias entre los tipos de apoyo y los distintos proveedores de apoyo (Clark-Lempers, Lempers y Ho, 1991; Procidiano y Heller, 1983).

En cuanto a la relación entre el apoyo social que perciben los estudiantes de sus compañeros/as y el clima social del centro escolar podemos observar que se relacionan positivamente con el aumento del clima social en las dos dimensiones de clima social, en relación al centro y en relación a los profesores. Este hallazgo se observa para el alumnado inmigrante y el alumnado autóctono pero no se han obtenido correlaciones significativas para el apoyo del alumnado de etnia gitana y el clima social. Por otro lado, el aumento de la frecuencia y satisfacción con el apoyo social que proporciona el profesorado, también se relaciona positivamente con el aumento del clima social en las dos dimensiones, lo que viene a confirmar la función importante que tiene el profesorado como fuente de apoyo en el contexto escolar. Estos resultados concuerdan con otros estudios que confirman que las relaciones positivas con el profesorado se relacionan con una mejor adaptación a la escuela (Cemalcilar, 2010; Crosnoe, Johnson y Elder, 2004)

Respecto a la relación entre el apoyo social de los estudiantes con los problemas de convivencia en el aula y con los conflictos debidos a las diferencias culturales podemos concluir que estos problemas disminuyen conforme aumenta la percepción de apoyo social, especialmente entre el alumnado inmigrante y autóctono. Este hallazgo coincide con otras investigaciones que evidencian que la percepción de apoyo de los compañeros/as de clase se relaciona positivamente con menos conductas violentas y más conductas prosociales con los iguales (Battistich, Schaps y Wilson, 2004). En cuanto al apoyo del profesorado, los resultados son también interesantes ya que la percepción de apoyo social que tienen los estudiantes de sus profesores/as se relaciona con la disminución de los problemas de convivencia en el aula pero no con los conflictos debidos a las diferencias culturales, únicamente es la satisfacción con el apoyo social emocional del profesorado, el tipo de apoyo que se relaciona con la disminución de este tipo de conflictos. Es posible, que el profesorado ponga en marcha estrategias conocidas para proporcionar el apoyo que necesitan los estudiantes para resolver los problemas de convivencia habituales en el aula, pero los conflictos derivados de las diferencias étnicas son 
más recientes y específicos. Este hallazgo seguramente tiene que ver con que hasta épocas recientes en las aulas no existía la diversidad del alumnado que caracteriza hoy día al contexto escolar. En la actualidad, el profesorado necesita un asesoramiento especializado en el ámbito de la atención a la diversidad, donde adquieran pautas y estrategias de actuación para poder trabajar con un alumnado tan diverso y tan diferente como el que existe actualmente en nuestras aulas (Sánchez, 2002). La satisfacción con el apoyo emocional que proporciona el profesorado ha mostrado ser muy importante en la reducción de conflictos multiculturales, pero sin duda cada tipo de apoyo cumple con una función específica y es necesario que los profesores, como importantes fuentes de apoyo en el contexto escolar, también conozcan el tipo de apoyo informacional e instrumental necesario para resolver estos conflictos. En este sentido, algunos autores creen que es importante trabajar con el profesorado diferentes estrategias de apoyo social en el aula (Juvonen, Nishina y Grahan, 2006)

El alumnado de etnia gitana ha mostrado en nuestro estudio ser el grupo más desfavorecido y con menor percepción de apoyo social, por debajo de los alumnos inmigrantes, cuya llegada a nuestras aulas ha sido mucho más reciente. Además perciben que el apoyo que reciben procede de su propio colectivo, cosa que no ocurre con los inmigrantes que perciben un importante apoyo social de sus compañeros autóctonos. Esto sigue evidenciando la situación de marginación y escasa percepción de apoyo en la que se sigue encontrando este colectivo, como muestran la mayoría de los estudios (Derringhton, 2007; García, 2005; Garreta, 2003; Márquez y Padua, 2009; Navas y Cuadrado, 2003; Rodríguez-Bailón y Moya, 2003). Por ello, creemos que el fortalecimiento de redes sociales entre todos los grupos de estudiantes puede ser uno de los factores principales a trabajar con este grupo de población de cara a facilitarles una mayor integración. En el informe elaborado por la Fundación Secretariado General Gitano (2002) sobre la evaluación de la normalización educativa del alumnado gitano en educación primaria, se concluye que la mayoría del alumnado gitano recibe menos elecciones para jugar o trabajar y más rechazos para este tipo de actividades que los alumnos del grupo mayoritario, aunque hay un pequeño grupo, alrededor del $27 \%$, que se encuentra en una situación normalizada.

Los resultados de nuestro estudio vienen a confirmar la necesidad de analizar las diferentes fuentes de apoyo en el contexto escolar, para conocer mejor la experiencia real del apoyo y la importancia de estudiar el apoyo social desde la perspectiva de las distintas fuentes y los diferentes contextos, como indican los modelos ecológicos y sistémicos (Bronfenbrenner, 2005). En el contexto escolar el apoyo social proporcionado por el profesorado y los compañeros/as de clase se asocia con un adecuado desarrollo emocional (Galanaki, Polychronopoulou y Babalis, 2008). También investigaciones recientes han mostrado que el apoyo social que proviene del contexto escolar tiene un importante papel en la integración de los adolescentes inmigrantes y las minorías étnicas (Crockett et al., 2007; Walsh, Harel-Fisch y FogelGrinvald, 2010). Los estudiantes inmigrantes además de enfrentarse a los cambios propios de la adolescencia se enfrentan a todos los eventos estresantes que acompañan al fenómeno de la inmigración, como la pérdida de redes sociales y los cambios en la estructura familiar. En esta situación, la capacidad de los padres para prestar apoyo disminuye y la escuela tiene la oportunidad de jugar un papel muy importante como fuente proveedora de apoyo social (Portes, 1997). También los hallazgos de este trabajo coinciden con otros estudios que destacan el importante papel que hoy día tiene la escuela en un contexto multicultural contribuyendo al bienestar de los inmigrantes adolescentes (Le, Lai y Wallen, 2009); mejorando las relaciones intergrupales (Graham, 2006) y fomentando la tolerancia y la aceptación de otros puntos de vista (kurlaender y Yun, 2002)

Es necesario también señalar la existencia de algunas limitaciones en la presente investigación. En este sentido, la utilización de autoinformes en adolescentes puede constituir una cierta limitación. Aunque este tipo de medición ha demostrado su validez, y de hecho constituye una fuente básica de información en estas investigaciones (Crick y Bigbee, 1998). Por otra parte, y debido a la naturaleza transversal de este estudio, los resultados deben interpretarse con cierta cautela, puesto que este tipo de trabajos no permiten establecer relaciones de causalidad. En posteriores estudios sería conveniente emplear una metodología longitudinal que permita analizar con mayor profundidad el modo en que estas variables evolucionan en los tres grupos de estudiantes analizados. También sería interesante, a pesar de la dificultad, aumentar el número de participantes de étnia gitana. No obstante, y teniendo en cuenta estas limitaciones las aportaciones de este estudio al ámbito educativo destacan la importancia de conocer y potenciar las redes sociales e interpersonales de los estudiantes y la necesidad de favorecer situaciones que ayuden al alumnado a interaccionar con su grupo de clase, independientemente de su origen o cultura, de manera que se creen lazos afectivos y emocionales entre ellos. El contacto con diferentes redes sociales contribuye a la integración social. Como se ha podido observar, la percepción de apoyo social por parte de compañeros / as y el profesorado repercute en una mejora del clima social del centro escolar y en la reducción de conflictos, este dato es muy importante para generar estrategias de intervención orientadas a incrementar las relaciones sociales positivas entre las diferentes fuentes de apoyo del contexto escolar.

Por otro lado, debemos formar e informar a todos los miembros de la comunidad educativa (profesorado, alumnado y familias) acerca de la realidad existente en nuestras aulas, basada en la diversidad. Se han de conocer términos como Atención a la Diversidad para posteriormente trabajar por una educación intercultural. En la nueva sociedad plural el establecimiento de lazos sociales y la cooperación son elementos importantes para prevenir los problemas que pudieran surgir. Además es importante optimizar todas las posibi- 
lidades individuales y sociales que ofrece el multiculturalismo (Martínez-Otero, 2003).

\section{Referencias}

Abajo, J.E. (2004). Infancia gitana y paya: Convivencia y conflictos en la escuela. Tabanque, 18, 97-116.

Aguado, T. (2003). Pedagogía intercultural. Madrid: McGraw-Hill Interamericana de España.

Andrés, M.T. (2006). La comunidad gitana y la educación. Madrid: FSG.

Antrop-González, R. (2006). Toward the school as sanctuary concept in multicultural urban education: Implications for small high school reform. Curriculum Inquiry, 36, 273-301.

Azmitia, M., Cooper, C.R. y Brown, J.R. (2009). Support and guidance from families, friends, and teachers in Latino early adolescent's math pathways. The Journal of Early Adolescence, 29, 142-169.

Barrón, A. (1996). El apoyo social. Aspectos teóricos y aplicaciones. Madrid: Siglo XXI.

Bartolomé, M. (2002). Identidad y ciudadanía. Un reto a la educación intercultural. Madrid: Narcea.

Battistich, V., Schaps, E. y Wilson, N. (2004). Effects of an elementary school intervention on students' "connectedness" to school and social adjustment during middle school. The Journal of Primary Prevention, 24, 243-262.

Beale, A.V. y Scott, P.C. (2001). "Bullybusters": Using drama to empower students to take a stand against bullying behavior. Professional School Counseling, 4, 300-305.

Berndt, T.J. y Savin-Williams, R.C. (1993). Peer relations and friendships. En P.H. Tolan y B. Cohler (Eds.), Handbook of clinical research and practice with adolescents (pp. 203-219). Nueva York: John Wiley \& Sons.

Bernstein, J.Y. y Watson, M.W. (1997). Children who are targets of bullying: A victim pattern. Journal of Interpersonal Violence, 12, 483-498.

Bronfenbrenner, U. (1979). Contexts of child rearing: Problems and prospects. American Psychologist, 34, 844-850.

Bronfenbrenner, U. (1995). Developmental ecology through space and time: A future perspective. En P. Moen, G.H. Elder y K. Luscher (Eds.), Examining lives in context: Perspectives on the ecology of human development (pp. 619-647). Washington, DC: American Psychological Association.

Bronfenbrenner, U. (2005). Making human beings human: Bioecological perspectives on buman development. Thousand Oaks, CA: Sage.

Cangas, A.J., Gázquez, J.J., Pérez-Fuentes, M.C., Padilla, D. y Miras, F. (2007). La evaluación de la violencia escolar y su afectación personal en una muestra de estudiantes europeos. Psicothema, 19, 114-119.

Caplan, G. (1974). Support systems and community mental health: Lectures on concept development. Nueva York: Behavioral Publications.

Cava, M. J. (2011). Familia, profesorado e iguales: Claves para el apoyo a las víctimas de acoso escolar. Psychosocial Intervention, 20, 183-192.

Cemalcilar, Z. (2010). Schools as socialization contexts: Understanding the impact of school climate factors on students' sense of school belonging. Applied Psychology: An International Review, 59, 243-272.

Centro de Investigación y Documentación Educativa. (2007). Evolución y situación actual de la presencia del alumnado extranjero en el sistema educativo español (1996-2007). Madrid: Ministerio de Educación y Ciencia.

Clark-Lempers, D.S., Lempers, J.D. y Ho, C. (1991). Early, middle and late adolescents' perceptions of their relationships with significant other. Journal of Adolescent Research, 6, 296-315.

Cohen, S. y Mckay, G. (1984). Social support, stress and the buffering hypothesis: A theoretical analysis. En A. Baum, S.E. Taylor y J.E. Singer (Eds.), Handbook of Psychology and Health (pp. 253-267). Hillsdale, N.J: Lawrence Erlbaum.

Cohen, S. y Wills, T.A. (1985). Stress, social support, and the buffering hypothesis. Psychological Bulletin, 98, 310-357.

Coie, J.D. y Dogde, K.A. (1998). Aggresion and antisocial behavior. En W. Damon (Ed. Serie), y N. Eisenberg (Ed.Vol), Handbook of Child Psychology. Vol.3: Social, emotional and personality development (pp.779-862). Nueva York: Wiley.
Agradecimientos.- Esta investigación forma parte de otro proyecto más amplio financiado por el Ministerio de Ciencia y Tecnología (BSO2002-03460) con Fondos Feder y por la Consejería de Gobernación. Dirección General de Políticas Migratorias (Ref. 2008/99). Junta de Andalucía (España).

Cook, T.D., Herman, M.R., Phillips, M. y Settersten, R.A. (2002). Some ways in which neighborhoods, nuclear families, friendship groups, and schools jointly affect changes in early adolescent development. Child Development, 73, 1283-1309.

Crick, N. R. y Bigbee, M. A. (1998). Relational and overt forms of peer victimitation:

A multi- informant approach. Journal of Consulting and Clinical Psychology, 66, 337- 347 .

Crockett, L.J., Iturbide, M.I., Torres Stone, R.A., McGinley, M., Raffaelli, M. y Carlo, G. (2007). Acculturative stress, social support, and coping: Relations to psychological adjustment among Mexican American college students. Cultural Diversity and Ethnic Minority Psychology, 13, 347-355.

Crosnoe, R., Johnson, M.K. y Elder, G.H, Jr. (2004). Intergenerational bonding in school: The behavioral and contextual correlates of studentteacher relationships. Sociology of Education, 77, 60-81.

Del Valle, J., Bravo, A. y López, M. (2010). Parents and peers as providers of support in adolescents'social network: a developmental perspective. Journal of Community Psychology, 38, 16-27.

Demaray, M.K. y Malecki, C.K. (2002). The relationship between perceived social support and maladjustment for students at risk. Psychology in the Schools, 39, 305-316.

Derringhton, Ch. (2007). Fight, flight and playing white: An examination of coping strategies adopted by gipsy traveler adolescents in english secondary schools. International Journal of Educational Research, 46, 357-367.

Díaz-Aguado, M.J. (2003). Educación intercultural y aprendizaje cooperativo. Madrid: Pirámide.

Domínguez, J.M y Hombrados, M.I. (2008). Happiness in immigrant women in Spain. Journal of Immigrant \&o Refugee Studies, 6, 267-273.

Durkheim, E. (1976). El suicidio. Madrid: Akal.

Eccles, J.S. y Roeser, R.W. (2003). Schools as developmental contexts. En G. Adams y M.D. Berzonsky (Eds.), Blackwell Handbook of Adolescence (pp.129-148). Malden, MA: Blackwell Publishing.

Emmons, C.L., Comer, J.P. y Haynes, N.M. (1996). Translating theory into practice: Comer's theory of school reform. En J.P. Comer, N.M Haynes, E. Joyner y M. Ben-Avie (Eds.), Rallying the whole village (pp. $27-$ 41). Nueva York: Teachers College Press.

Essomba, M.A. (2007). Estrategias de innovación para construir la escuela intercultural. En J.L. Álvarez y L. Batanaz (Eds.), Educación intercultural e inmigración. De la teoría a la práctica (pp. 177-212). Madrid: Biblioteca Nueva.

Esteve, J.M. (2003). La tercera revolución educativa. La educación en la sociedad del conocimiento. Barcelona: Paidós.

Farrington, D.P. (1993). Understanding and preventing bullying. En M. Tonry (Ed.), Crime and Justice (pp. 381-458). Chicago: University Chicago Press.

Felner, R.D., Brand, S., Dubois, D.L., Adan, A.M., Mulhall, P.F. y Evans, E.G. (1995). Socioeconomic disadvantage, proximal environmental experiences and socio-emotional and academic adjustment in early adolescence: investigation of a mediated effects model. Child Development, 66, 774-792.

Fernández, F.J., Trianes, M.V., de la Morena, L., Escobar, M., Infante, L. y Blanca, M.J. (2011). Propiedades psicométricas de un cuestionario para la evaluación de la violencia cotidiana entre iguales en el contexto escolar. Anales de Psicología, 27, 102-108.

Fernández, J.S. y Soriano, E. (1996). Sociometría y etnicidad: realidad multicultural e integración en las escuelas almerienses. V Conferencia de Sociología de la Educación. Universidad Rovira i Virgil, Tarragona.

Franco, N. y Levitt, M.J. (1998). The social ecology of middle childhood: Family support, friendship quality, and self-esteem. Family Relations, 47, 315-321. 
Fundación Secretariado General Gitano. (2002). Evaluación de la Normalización Educativa del alumnado gitano en Educación Primaria. Madrid: FSGG.

Furman, W. y Buhrmester, D. (1992). Age and sex differences in perceptions of networks of personal relationships. Child Development, 63, 103-115.

Galanaki, E.P., Polychronopoulou, S.A. y Babalis, T.K. (2008). Loneliness and social dissatisfaction among behaviourally at-risk children. School Psychology International, 29, 214-229.

García, A. (2005). La educación con niños gitanos. Una propuesta para su inclusión en la escuela. Revista Electrónica Iberoamericana sobre Calidad, Eficacia y Cambio en Educación, 3 (1), 437-448.

Garnefski, N. y Diekstra, R.F.W. (1996). Perceived social support from family, school and peers: Relationship with emotional and behavioral problems among adolescents. Journal of the American Academy of Child and Adolescent Psychiatry, 35, 1657-1664.

Garreta, J. (2003). La integración sociocultural de las minorías étnicas (gitanos e inmigrantes). Barcelona: Anthropos Editorial.

Giménez, C. y Pérez, C. (2003). La integración de los menores de origen extranjero en la Comunidad de Madrid: necesidades, derechos y actuaciones. En Asamblea de Madrid y Defensor del Menor: Estudios e Investigaciones 2002. Defensor del menor de la Comunidad de Madrid.

Gracia, E. (1997). El apoyo social en la intervención comunitaria. Barcelona: Paidos.

Gracia, E. y Herrero, J. (2004). Personal and situational determinants of relationship-specific perceptions of social support. Social Behavior and Personality, 32, 459-476.

Graham, S. (2006). Peer victimization in school: Exploring the ethnic context. Current Directions in Psychological Science, 15, 317-321.

Greve, W. y Wilmers, N. (2003). School violence and self-esteem: Moderating influences of coping resources among offenders and victims. Psychologic in Erziehung und Unterricht, 50, 353-368.

Hall, A. y Wellman, B. (1985). Social networks and social support. En S. Cohen y S. Syme (Eds.), Social support and health (pp.23-41). Nueva York: Academic Press.

Hao, L. (2003). Private Support and Public Assistance for Immigrant Families. Journal of Marriage and Family, 65, 36-51.

Harknett, K. (2006). The relationship between private safety nets and economic outcomes among single mothers. Journal of Marriage and Family, $68,172-191$.

Harter, S. (1985). Manual for the social support scale for children. Denver, CO: University of Denver.

Henley, J.R., Danziger, S.K. y Offer, S. (2005). The contribution of social support to the material well-being of low-income families. Journal of Marriage and Family, 67, 122-140.

Herrero, J., Fuente, A. y Gracia, E. (2011). Covariates of subjective wellbeing among Latin American immigrants in Spain: the role of social integration in the community. Journal of Community Psychology, 39, 761-775.

Herrero, J. y Gracia, E. (2007). Measuring perceived community support: Factorial structure, longitudinal invariance, and predictive validity of the PCSQ (perceived community support questionnaire). Journal of Community Psychology, 35, 197-217.

Hombrados, M.I., García, M.A. y Moscato, G. (2005). Senso di comunitá e partecipacione sociale nei consigli comunali per minori. Psicología di Comunitá, 2, 101-117.

Johns, S.E. (2001). Using the comer model to educate immigrant children. Childhood Education, 77, 268-274.

Jordán, J.A. (1999): El profesorado ante la educación intercultural. En M.A. Essomba (Ed.), Construir la escuela intercultural. Reflexiones y propuestas para trabajar la diversidad étnica y cultural (pp.65-73). Barcelona: Grao.

Jordán, J.A. (2007). Educar en la convivencia en contextos multiculturales. En E. Soriano (Ed.), Educación para la convivencia intercultural (pp. 59-94). Madrid: La Muralla.

Juvonen, J., Nishina, A. y Graham, S. (2006). Ethnic diversity and perceptions of safety in urban middle schools. Psychological Science, 17, 393-400.

Kim, H.K. y McKenry, P.C. (1998). Social networks and support: A comparison of African Americans, Asian Americans, Caucasians and Hispanics. Journal of Comparative Family Studies 29, 313-334.

Kuperminc, G.P., Blatt, S.J. y Leadbeater, B.J. (1997). Relatedness, selfdefinition and early adolescent adjustment. Cognitive Therapy and Research, $21,59-78$.
Kurlaender, M. y Yun, J.T. (2002). The impact of racial and ethnic diversity on educational outcomes; Cambrigde, MA School District. Cambrigde, MA: The Civil Rights Project, Harvard University.

Ladd, G.W. (1990). Having friends, keeping friends, making friends, and being like by peers in the classroom: predictors of children's early school adjustment. Child Development, 61, 312-331.

La Greca, A.M. y López, N. (1998). Social anxiety among adolescents: Linkages with peer relations and friendships. Journal of Abnormal Child Psychology, 26, 83-94.

Laireiter, A. y Baumann, U. (1992). Network structures and support functions: Theoretical and empirical analyses. En H.O. Veiel y U. Baumann (Eds.), The meaning and measurement of social support (pp. 33-35). Nueva York: Hemisphere.

Leiva, J. (2007). Educación y conflicto en escuelas interculturales. Málaga: Spicum.

León, M.J. (2008). El reto de educar en una sociedad plural. Educatio Siglo XXI, 26, 161-178.

Le, T.N., Lai, M.H. y Wallen, J. (2009). Multiculturalism and subjective happiness as mediated by cultural and relational variables. Cultural Diversity and Ethnic Minority Psychology, 15, 303-313.

Levitt, M.J. (2005). Social relations in childhood and adolescence: The convoy model perspective. Human Development, 48, 28-47.

Lin, N. (1986). Conceptualizing social support. En N. Lin, A. Dean y W.N. Ensel (Eds.), Social support, life events and depression (pp. 17-30). Nueva York: Academic Press.

Lin, N. (2001). Social capital: a theory of social structure and action. Cambrigde, UK: Cambrigde University Press.

Lin, N. y Ensel, W. (1989). Life stress and health: Stressors and resources. American Sociological Review, 54, 382-399.

López, M. (2006). Cultura de la diversidad, cultura de la inclusión: educar para construir una escuela sin exclusiones. En Actas de las XVI Jornadas Municipales de Psicopedagogía L'Ecola que inclou" (pp. 11-52). Ajuntament de Torrent: Colecció Hort de Trenor.

Malecki, C.K. y Demaray, M.K. (2002). Measuring perceived social support: development of the child and adolescent social support scale (CASS). Psychology in the Schools, 39, 1-18.

Malecki, C.K. y Demaray, M.K. (2003). What type of support do they need? Investigating student adjustment as related to emotional, informational, appraisal and instrumental support. School Psychology Quarterly,18, 231252.

Márquez, M.J. y Padua, D. (2009). La institución educativa, un espacio a revisar: las adolescentes gitanas en su trayectoria educativa. Revista Interuniversitaria de Formación del Profesorado, 64, 73-88.

Martínez-Otero, V. (2003). Educación intercultural y desarrollo de la personalidad. Educación y futuro: revista de investigación aplicada y experiencias educativas, 8, 51-60.

Martínez, M.F., García, M. y Maya, I. (1999). El papel de los recursos sociales naturales en el proceso migratorio. Intervención Psicosocial, 8, 221-232.

Martínez, F., Moreno, D., Amador, L. V.y Orford, J. (2011). School victimization among adolescents. Analysis from an ecological perspective. Psychosocial Intervention, 20, 149-160.

Ministerio de Educación. (2009). Datos y Cifras. Curso escolar 2010-2011. Madrid: Secretaría General Técnica. Catálogo de publicaciones del Ministerio de Educación.

Musitu, G., Buelga, S., Lila, M. y Cava, M.J. (2001). Familia y adolescencia. Madrid: Síntesis.

Musitu, G. y Cava, M.J. (2003). El rol del apoyo social en el ajuste de los adolescentes. Intervención Psicosocial, 12, 179-192.

Navas, M y Cuadrado, I. (2003). Actitudes hacia inmigrantes africanos y gitanos. Un estudio comparativo. Apuntes de Psicologia, 21, 29-49.

Navas, M., García, C., Rojas, A., Pumares, P. y Cuadrado, I. (2006). Actitudes de aculturación y prejuicio: la perspectiva de autóctonos e inmigrantes. Psicothema, 18, 187-193.

Observatorio Permanente Andaluz de las Migraciones (2010). Alumnado extranjero en el sistema educativo. Sevilla: Unidad de Estadística de la Consejería de Educación de la Junta de Andalucía.

Oppedal, B., Roysamb, E. y Lackland, D. (2004). The effect of acculturation and social support on change on mental health among young immigrants. International Journal of Behavioral Development, 28, 481-494. 
Parker, J.G. y Asher, S.R. (1987). Peer relations and later personal adjustment: Are low-accepted children at risk? Psychological Bulletin, 102, $357-$ 389.

Pellegrini, A.D., Bartini, M. y Brooks, F. (1999). School bullies, victims, and aggressive victims: Factors relating to group affiliation and victimization in early adolescence. Journal of Educational Psychology, 91, 216-224.

Phelan, P., Davidson, A.L. y Cao, H.T. (1991). Students' multiple worlds: Negotiating the boundaries of family, peer, and school cultures. Anthropology and Education Quarterly, 22, 224-250.

Phinney, J.S., Ong, A. y Madden, T. (2000). Cultural values and intergenerational value discrepancies in immigrant and non-immigrant families. Child Development, 71, 528-539.

Portes, A. (1997). Immigration theory for a new century: Some problems and opportunities. International Migration Review, 31, 799-825.

Procidiano, M.E. y Heller, K. (1983). Measures of perceived social support from friends and from family: Three validation studies. American Journal of Community Psychology, 11, 1-24.

Redondo, S. y Garrido, V. (2001). Violencia y delincuencia juvenil: Explicación y prevención. Mendoza: Ediciones Jurídicas Cuyo.

Robinson, N.S. (1995). Evaluating the nature of perceived support and its relation to perceived self-worth in adolescents. Journal of Research on Adolescence, 5, 253-280.

Rodriguez-Bailón, R. y Moya, I. (2003). La diferencia percibida en valores como proceso vinculado a las relaciones intergrupales de payos y gitanos. Psicothema, 15, 176-182.

Ryan, R., Kalil, A. y Leininger, L. (2009). Low-income mothers' private safety nets and children's socioemotional well-being. Journal of Marriage and Family, 71, 278-297.

Sánchez, A.M., Rivas, M.T. y Trianes, M.V. (2006). Eficacia de un programa de intervención para la mejora del clima escolar: Algunos resultados. Revista Electrónica de Investigación Psicoeducativa, 4, 353-370.

Sánchez Delgado, P. (2002). Atención a la diversidad cultural en los centros educativos. Propuestas de reflexión y acción sobre el proyecto educativo, el proyecto curricular y la práctica en el aula. Revista Complutense de Educación, 2, 677-706.

Santos, M.A. (2008). La pragmática de la cooperación o cómo mejorar la gestión de la diversidad cultural y de la convivencia en las aulas. En J. Vera (Ed.), Diversidad, convivencia y educación desde el conflicto (pp.55-75). Madrid: Fundación SM.

Sarason, I.G., Levine, H.M., Basham, R.B. y Sarason, B.R. (1983). Assessing social support: The social support questionnaire. Journal of Personality and Social Psychology, 44, 127-139.

Sarason, I.G., Pierce, G.R. y Sarason, B.R. (1990). Social support and interactional processes: A triadic hypothesis. Journal of Social and Personal Relationships, 7, 495-506.

Searle, W. y Ward, C. (1990). The prediction of psychological and sociocultural adjustment during cross-cultural transitions. International Journal of Intercultural Relations, 14, 449-464.
Seidman, E., Allen, L., Aber, J.L., Mitchel, C. y Feinman, J. (1994). The impact of school transitions in early adolescence on the self-system and perceived social context of poor urban youth. Child Development, 65, 507522.

Schaefer, C., Coyne, C. y Lazarus, R. (1981). The health-related functions of social support. Journal of Behavioral Medicine, 4, 381-406.

Siegler, R., Deloache, J. y Eisenberg, N. (2006). How children develop. Nueva York: Worth Publishers.

Slaby, R.G. y Roedell, W.C. (1982). Development and regulation of aggression in young children. En J. Worrell (Ed.), Psychological development in the elementary years (pp. 97-149). Nueva York: Academic Press.

Smetana, J.G., Campione-Barr, N. y Metzger, A. (2006). Adolescent development in interpersonal and societal contexts. Annual Review of Psychology, 57, 225-284.

Soriano, E. (2008). Formación del profesorado para la educación intercultural. En J. Vera (Ed.), Propuestas y experiencias de educación intercultural (pp.57-84). Madrid: Fundación SM.

Suarez-Orozco, M. (2001). Globalization, immigration, and education: The research agenda. Harvard Educational Review, 71, 345-365.

Taylor, S.E., Sherman, D.K., Kim, H.S., Jarcho, J., Takagi, K. y Dunagan, M.S. (2004). Culture and social support: Who seeks it and why? Journal of Personality and Social Psychology, 87, 354-362.

Trianes, M.V. (2000). Violencia en contextos escolares. Málaga: Aljibe.

Trianes, M.V., Blanca, M.J., De la Morena, L., Infante, L. y Raya, S. (2006). Un cuestionario para evaluar el clima social del centro escolar. Psicothema, 18, 272-277.

Turney, K. y Kao, G. (2009). Assessing the private safety net: Social support among minority immigrant parents. The Sociological Quarterly, 50, 666692.

Walsh, S.D., Harel-Fisch, Y. y Fogel-Grinvald, H. (2010). Parents, teachers and peer relations as predictors of risk behaviors and mental well-being among immigrant and Israeli born adolescents. Social Science and Medicine, 70, 976-984.

Westling, M. (2002). A two-level analysis of classroom climate in relation to social context, group composition and organization of special support. Learning Environments Research, 5, 253-274.

Wills, T.A. y Shinar, O. (2000). Measuring perceived and received socia support. En S. Cohen, L.G. Underwood y B.H. Gottlieb (Eds.), Social support measurement and intervention: A guide for health and social scientists (pp. 86-135). Oxford, UK: Oxford University Press.

Woolley, M.E., Kol, K. y Bowen, G.L. (2009). The social context of school success for Latino middle school students: Direct and indirect influences of teachers, family, and friends. Journal of Early Adolescence, 29, 4370

(Artículo recibido: 26-03-2011, revisión: 28-01-2012, aceptado: 30-01-2012) 